\title{
Networked Sensing Systems for Detecting People Carrying Radioactive Material
}

\author{
Mani Chandy, Concetta Pilotto and Ryan McLean \\ California Institute of Technology \\ Pasadena, CA 91125 \\ Email: mani@cs.caltech.edu
}

\begin{abstract}
The goal of the research described in this paper is to help prevent scenarios such as the following: a terrorist detonates a device that distributes radioactive material such as Cesium137 or Cobalt-60 in an open space used for public sports events or demonstrations. This paper studies the efficacy of networks of static sensors on street lamps or similar infrastructures. This paper describes individual sensors, evaluates the benefits of networks of stationary sensors, and briefly discusses the potential value of integrated networks of wireless-equipped mobile security personnel with stationary radiation sensors and cameras. The paper presents mathematical analysis coupled with simulation results.
\end{abstract}

Keywords - radiation detection; networking systems; sensors

\section{The Problem}

The goal of the research described in this paper is to help prevent scenarios such as the following: a terrorist walks in a crowd participating in a political demonstration in an open space, such as a football field, and detonates a device that disperses radioactive material. We study situations in which searching backpacks of all participants in the event is infeasible. We study systems with the following goals.

System Goal: A network of stationary sensors is required to inform security personnel when there is a radioactive source in the field. The sensors may be located on street lamps, utility devices such as telephone poles, or temporary traffic sign stands. The radiation source may be mobile or stationary. The system is required to detect the presence of radioactive sources rapidly, and identify their locations accurately.

The performance metrics of the system include the rate of false alarms, the time to detect a source, and the area in which the source is predicted to be located. We evaluate these performance metrics for different design parameters such as the number of sensors distributed over a region, the relative benefits of directional sensors focused on narrow beams versus omni-directional sensors, the effect of background radiation, and the speed and complexity of computation.

Background radiation is emitted from the ground, buildings and people. The amount of background radiation may vary with environmental conditions such as rain, location of vehicles, or presence of patients with benign medical sources, such as Technetium-99 or Iodine-131. An advantage of stationary sensors is that the background noise can be characterized accurately by making measurements over time in different conditions.
Terrorists may have access to different radioactive compounds such as Cesium-137 or Cobalt-60. In this paper, we do not investigate the problem of identifying isotopes from radiation energy signatures. Nor do we consider important issues such as the benefits of coupling stationary radiationdetection networks with surveillance cameras and mobile sensors on small unmanned aerial vehicles. The paper restricts attention to the benefits of networking sensors.

\section{Model of Sensors and Radioactive Sources}

Research on sensors for radiation detection has been carried out for decades. The early research was primarily for scientific experiments; the more recent work is also for security. At Caltech we are working towards handheld detectors coupled with wireless communication for problems such as those identified in the Intelligent Personal Radiation Locator (IPRL) program. This paper is motivated by laboratory experiments that suggest the possibility of deploying adequate-resolution gamma-ray detectors at room temperatures. Initial designs and laboratory experiments indicate that crystal detectors and associated electronics can be manufactured in large quantities at reasonable prices. This preliminary analysis leads to the question: Can networks of such sensors be cost effective in locating radiation?

We have developed advanced readouts for CdZnTe detectors (see figure 1). CdZnTe is a large bandgap semiconductor material that can be operated as a good resolution gamma-ray detector at room temperature. As such, it performs with higher resolution than a scintillating crystal, e.g. NaI, without the cryogenic cooling requirement of a high-purity Ge detector. The Caltech readouts are bonded directly to the back of $20 \mathrm{~mm} \times 20 \mathrm{~mm} \times 5 \mathrm{~mm}$ slabs of CdZnTe and pixelate the crystal into an array of $32 \times 32$ smaller detectors, each of which has less noise than a single readout of the full detector. The detectors and associated electronics are light, weighing less than a kilogram, and can be carried by small UAVs and even model airplanes. Figure shows photographs of the sensor. This paper, however, focuses on designs and analysis of sensor networks rather than on individual sensors.

A radiation source is treated as a point source. Though radiation material may be distributed within a backpack an approximation to a point source is adequate for scenarios studied in this paper. A radiation source generates photons 


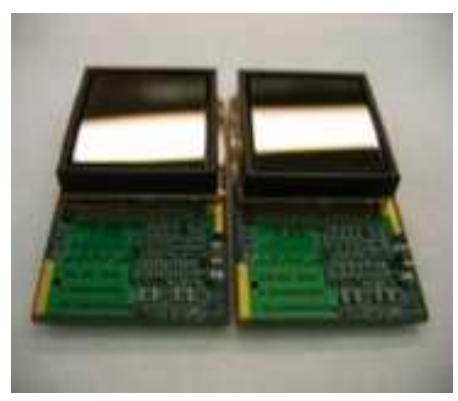

Fig. 1. IPRL handheld detectors developed at the California Institute of Technology.

in a Poisson manner at some rate $\mu$. A detector consists of a crystal coupled with electronics. When a photon interacts inside a crystal, the photon generates electron-hole pairs in proportion to the energy absorbed by the crystal. An electric field separates the charge carriers and the sensor measures the anode signal, which is mostly from the electrons. Measurements are imperfect; electron trapping and material flaws limit the resolution of the energy measurement and physics limits the number and quality of events in the crystal. A photon may be absorbed or scattered by material that it strikes. We begin by ignoring absorption and scattering; we consider these issues briefly later. Photons arrive at all points on a sphere of distance $r$ from the source with equal probability. As a first approximation, we treat the crystal as a flat plate. Therefore photons from a point source hit a stationary plate at a distance $r$ from the source in a Poisson process with rate $\lambda$ where:

$$
\lambda=\mu \cdot \cos \theta / r^{2}
$$

where $\theta$ is the angle between the normal to the plate and the line joining the plate and the source.

In addition to the geometrical effect above, there are several possible techniques for understanding the direction of the incoming photon. These include, collimation or shielding, Compton imaging and coded aperture imaging.

\section{DESIGN ISSUES}

The problem of detecting and interdicting a pedestrian carrying radioactive material has different aspects depending upon whether the person is walking along a sidewalk in a city, or within a building or within an open area such as a park. This paper considers only the open area scenario. Next, we outline the design issues studied in this paper.

Number of sensors: We study the benefits of increasing the number of sensors. In situations where there are few utility poles on which to place sensors, we study the effects of placing multiple directional sensors facing different regions of the field.

A priori estimates of target location and source intensity: We use Bayesian statistics to estimate the a posteriori location and intensity of a target given an a priori distribution and readings from each sensor over a time period. We study the effect of Bayesian updates without prior distributions on source intensity, and the benefits of accurate prior estimates.

Estimating area within which the target is located: The stationary sensor network can alert security personnel about a potential target identified to be within a large area (say 100 meter square) with fewer measurements and less time than predicting target location with pinpoint accuracy (say 1 meter square). We explore the relationship between estimated probability and the area within which a source is estimated to be located.

Effect of noise: The ground and buildings emit some radiation. The impact of this noise on stationary sensors can be factored out to a large extent but not eliminated. We study the relationship between noise and accuracy.

Most of this paper is concerned with interdicting a single source of radiation. We also briefly consider the problem of detecting multiple sources.

\section{AnAlysis}

\section{A. Sensor Network and Known Source}

In this section we assume the existence of a single source that generates photons with intensity $\mu$ at a point with coordinates $[x, y]$. We assume in this section, and this section only, that we know the source intensity $\mu$ and location $[x, y]$. Our problem is, of course, to estimate the source location and intensity.

Consider a region with $D$ sensors indexed $j$ where $1 \leq$ $j \leq D$. Photons from the source strike each of the sensors in an independent Poisson manner. Let $\lambda_{j}$ be the rate at which photons strike sensor $j$. To begin with assume that at all noise has been factored out. Also assume there is no absorption or scattering. The relationship between source intensity and the rate at which photons strike a sensor was given earlier in eqn.( 1).

Let $f(\lambda, n, T)$ be the probability of $n$ events (photons) in an interval of duration $T$ in a Poisson process with rate $\lambda$.

$$
f(\lambda, n, T)=\frac{(\lambda \cdot T)^{n} \cdot e^{-\lambda \cdot T}}{n !}
$$

Let $\vec{n}=\left[n_{1}, \ldots, n_{D}\right]$ and let $p(\vec{n}, T)$ be the probability that $n_{j}$ photons strike sensor $j$, for all $j$, in a time interval of duration $T$. Since the processes by which photons strike each of the sensors are mutually independent and are Poisson

$$
p(\vec{n}, T)=\prod_{j=1}^{D} f\left(\lambda_{j}, n_{j}, T\right)
$$

Let $N$ be the total number of photons to hit all the $D$ sensors in an interval when $n_{j}$ photons hit sensor $j$ :

$$
N=\sum_{j=1}^{D} n_{j}
$$


Let $\Lambda$ be the total intensity of photons on all the $D$ sensors:

$$
\Lambda=\sum_{j=1}^{D} \lambda_{j}
$$

Let $p_{j}$ be the probability that a photon that hits any one of the $D$ sensors hits sensor $j$.

$$
p_{j}=\frac{\lambda_{j}}{\Lambda}
$$

Let $g(\vec{n}, N)$ be the conditional probability that $n_{j}$ photons hit sensor $j$, all $j$, given that a total of $N$ photons hit all the $D$ sensors. Since each photon is independent of others, the conditional probability is a multionomial:

$$
g(\vec{n}, N)=N ! . \prod_{j=1}^{D}\left(p_{j}^{n_{j}} / n_{j} !\right)
$$

Manipulating eqn.( 2) we get $p(\vec{n}, T)$ to be the product of (i) the probability of a total of $N$ photons hitting all the sensors and (ii) the conditional probability that $n_{j}$ photons hit sensor $j$ given that a total of $N$ photons hit all $D$ sensors.

$$
p(\vec{n}, T)=f(\Lambda, N, T) \cdot g(\vec{n}, N)
$$

\section{B. Sensor Network and Unknown Single Source}

Consider the case where there exists exactly one source. The intensity and location of the source are unknown. Assume, to begin with, that sensors are spheres; hence, they have no directional preference and so the intensity with which photons from a source hits the sensor depends only on the distance from the sensor and not on the orientation of the sensor.

Let $d_{j}[x, y]$ be the distance from a point $[x, y]$ to sensor $j$. Let $p_{j}[x, y]$ be the conditional probability that a photon from a source at location $[x, y]$ hits sensor $j$ given that a photon from a source at this location his one of the $D$ sensors. Then from the earlier analysis - see eqn.( 1) and eqn.( 3 )

$$
p_{j}[x, y] \propto 1 / d_{j}[x, y]^{2}
$$

where the proportionality constant makes the sum of probabilities equal to unity. Hereafter, we wont mention the proportionality constant where it is obvious.

We study two algorithms for estimating the location and intensity of the source. (a) We analyze an algorithm that operates in two steps: the first step uses eqn.( 6) (which depends only on the location of the source and is independent of the intensity) to estimate the location of the source using Bayes law given an a priori distribution of location. The second step estimates the intensity given the probable location estimated in the first step. (b) We analyze an algorithm that estimates both location and intensity together given independent a priori distributions on both location and intensity.
We generate a grid over the region of interest with all elements of the grid being squares of equal size. We assume that sources, if any, are located at the center of a grid element. Let $q[x, y]$ be the a priori probability that a single source is at grid position $[x, y]$. The measured data is a vector of numbers of photons at each sensor $\vec{n}$ in a time interval of some duration $T$. Let $r[x, y]$ be the a posteriori probability given these measurements. Then,

$$
r[x, y] \propto q[x, y] . g(\vec{n}, N)
$$

where $p_{j}[x, y]$ replaces $p_{j}$ in $g$. This expression for $r[x, y]$ is equivalent to:

$$
r[x, y] \propto q[x, y] \cdot \prod_{j} p_{j}[x, y]^{n_{j}}
$$

We compute a posteriori distribution after a single photon hits a sensor, say sensor $j$, to get:

$$
r[x, y] \propto q[x, y] \cdot p_{j}[x, y]
$$

The values $p_{j}[x, y]$ are precomputed, and hence computation of the posteriori distribution with $G$ elements of the grid requires only $2 G$ multiplications and $G$ additions, resulting in small computation times. Computation times can be reduced further by ignoring regions where $q[x, y]$ and $p_{j}[x, y]$ are small; our experiments show that most of the grid can be ignored after just a few steps.

After the location is estimated, the intensity is estimated using a maximum likelihood estimator. From eqn.( 1) we see that the intensity $\lambda_{j}$ with which photons strike sensor $j$ is directly proportional to the intensity $\mu$ of the source. Hence the total intensity $\Lambda$ with which photons strike all the sensors is also proportional to $\mu$. Let

$$
\mu=\Lambda . A
$$

where $A$ is a constant of proportionality which is computed assuming that the source is located at the position estimated by the algorithm in the first step. Let $\hat{\mu}$ be our estimate of $\mu$. Then

$$
\hat{\mu}=(N / T) \cdot A
$$

Similar calculations can be carried out for the case where an a priori distribution over the source intensity $\mu$ is given to obtain:

$$
r[x, y, \mu]=q[x, y, \mu] \cdot \lambda_{j}[x, y, \mu]^{n_{j}} \cdot e^{-\lambda_{j} \cdot T}
$$

where now $r$ and $q$ include the source intensity $\mu$ in the a priori and posteriori distributions, and $\lambda_{j}[x, y, \mu]^{n_{j}}$ is the intensity with which photons strike sensor $j$ given a source with intensity $\mu$ at location $[x, y]$. Here too, most of the parameters can be precalculated; for example $\lambda_{j}[x, y, \mu]$ can be calculated for discrete values of $\mu$ and a range of $n_{j}$. Thus the posteriori distribution is computed rapidly. 


\section{Effect of Noise}

The background noise-photons generated by radioactivity in the ground and surroundings - can be estimated accurately for stationary sensors provided measurements can be made at each sensor in environmental conditions (such as rain or snow) that hold in the actual situation. We can estimate the location and intensity of a source by incorporating background noise into the a priori probability distribution. An approximation is to carry out calculations ignoring the noise; this approach can work because the effects of noise should cancel out provided there are enough sensors. An alternate approach is to assume a background rate $\gamma_{j}$ of photons hitting sensor $j$. Experiments are given in the next section.

\section{Multiple Sources and Motion Dynamics}

Multiple sources of radiation may represent innocent people who, for example, have ingested radioactive isotopes for medical purposes. Multiple sources may also represent multiple terrorists. The a priori probability distribution with $R$ sources has to consider the location and intensity of each source, resulting in a distribution with $(G \cdot M)^{R}$ values where $G$ is the number of elements in the location grid and $M$ is the number of possible values of the source intensity. With $G=10^{6}$ and $M=10$, we see that the number of variables in the distributions is $10^{14}$ with even two sources, and the direct Bayesian approach is intractable with three or more sources. A feasible approach uses an approximation based on the following analysis.

A source generates photons that strike sensors nearby and are unlikely to strike sensors far away because of the $1 / r^{2}$ effect. Our analysis and experiments show that we can safely assume that no source is further away than a distance $d$ of any sensor that has detected photons at rates equal to or lower than background noise rates, where the parameter $d$ depends on the intensity of the source. This allows us to reduce computations to grids of tractable size; an estimation step may calculate the location and intensity of possible sources in multiple (possibly overlapping) regions. The results reported here for single sources suggest how multiple sources can be estimated efficiently.

A terrorist may want to move towards a political leader or a stage or some other destination. Given a distribution of location and intensity at a time $t$, we first compute the distribution at a time $t+\tau$, for a time step $\tau$ by assuming some dynamics of motion of the source; the simplest case is that a source moves a distance of at most $v . \tau$ where $v$ is the maximum velocity of a pedestrian. These calculations are similar to calculations that have been studied extensively in target tracking. The difference is the in the equations used for filtering.

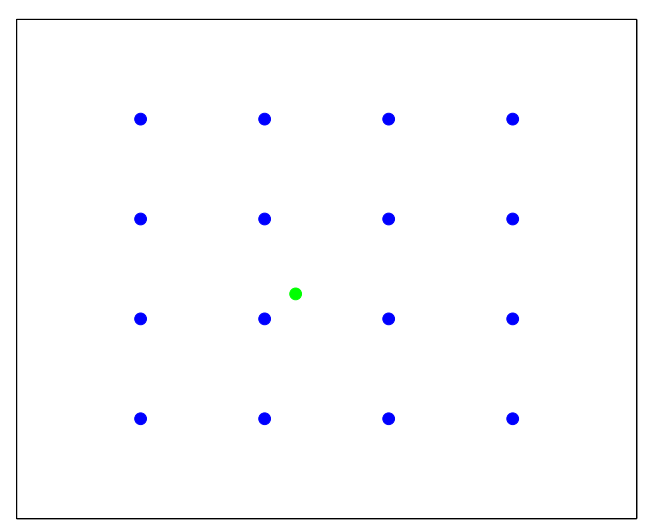

Fig. 2. Network configuration. Detectors are the blue circles. The source is the green circle.

\section{NUMERICAL RESULTS}

\section{A. Methodology}

The first set of numerical results are calculated for a $500 \mathrm{~m} \times 500 \mathrm{~m}$ area, shown in figure 2 with sixteen sensors (shown as blue circles in the figure) arranged in a uniform grid with a distance of $100 \mathrm{~m}$ between sensors. A single source, shown in the figure as a green circle, is located at the coordinates $(225,225) \mathrm{m}$. We consider spherical sensors of a fixed size; the spherical structure allows us to study systems without considering directionality. The intensity of the source is such that a sensor a meter away receives photons at a rate of 180 per second. This experiment represents IPRL detectors and a Cs137 0.6 milliCurie source. The first set of experiments assumes no background radiation.

Computations are carried out periodically with a period of 2 seconds using the equations given earlier. The area is partitioned into a grid of $100 \times 100$ square grid elements. We assume that if a source is in a grid element then it is located at the center of a grid element. We show contour plots of the estimate of the probability distribution of the source position; lighter colors are associated with unlikely source positions $(<0.0001)$, while darker colors represent more likely source positions $(>0.1)$. The plots are shown for the average of 100 runs. The estimates vary from one simulation to the next because the number of photons that hit each sensor vary randomly.

\section{B. Predictions with and without Assumptions About Source Intensity}

We present the simulation results for two different set of experiments.

a) Predictions without Assumptions About Source Intensity: For the first set of experiments, we update the estimated position of the source using the algorithm (a) of section IV which makes no assumptions about the emission 


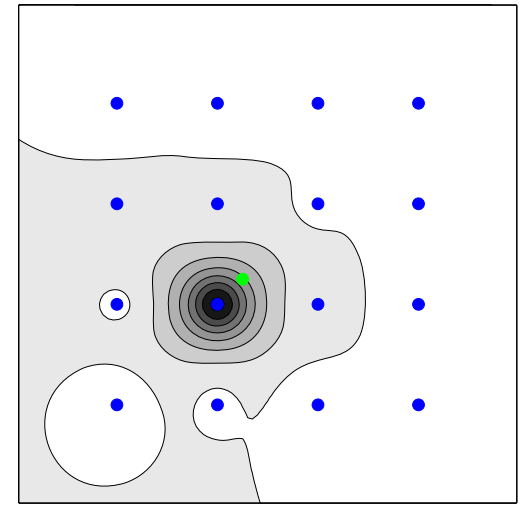

Fig. 3. The snapshot of the probability distribution for the non-parametric update algorithm after two seconds of computation.

rate of the source. Figures 3 and 4 show contour plots of a posteriori densities of the position of a source after 2 seconds and 10 seconds (respectively) of data. The sensor network correctly estimates that there is a radiation source in a $100 \mathrm{~m} \times 100 \mathrm{~m}$ square region centered around the sensor at coordinate $(200 \mathrm{~m}, 200 \mathrm{~m})$. The network estimates that a source falls within the region with probability 0.5491 after 2 seconds and probability 0.8732 after 10 seconds.

b) Predictions without Assumptions About Source Intensity: In the second set of experiments we assume the following a-priori probability distribution on the emission rate $\mu$ of the source: $\mu$ takes on the following multiples of the rate assumed in the earlier experiment $0.25,0.5,1,2,4$ with equal probability. This a-priori distribution is not accurate since it ranges uniformly over an interval whose maximum value is 16 times its minimum value. The quality of the estimates after 2 seconds depends critically upon the quality of the $a$ priori distribution; a bad a priori distribution is worse than no distribution at all. Nevertheless, the a posteriori distributions are accurate within 10 seconds. This set of experiments uses algorithm (b), which we call "intensity update algorithm". Figures 5 and 6 show contour plots of a posteriori densities of the position of a source after 2 seconds and 10 seconds (respectively) of data. The network estimates that the source lies in a $100 \mathrm{~m} \times 100 \mathrm{~m}$ square region centered around the sensor at coordinate $(200 \mathrm{~m}, 200 \mathrm{~m})$ with probability 0.3536 after 2 seconds and probability 0.9034 after 10 seconds.

\section{Background Noise}

The ground and buildings emit photons. Sophisticated sensors can differentiate between photons generated by materials that may be used to build dirty bombs from photons emitted by the ground. We consider the case where the background generates photons that are indistinguishable from photons from dangerous sources. We assume that each square in the grid emits photons in a Poisson manner at a rate of 0.3 photons

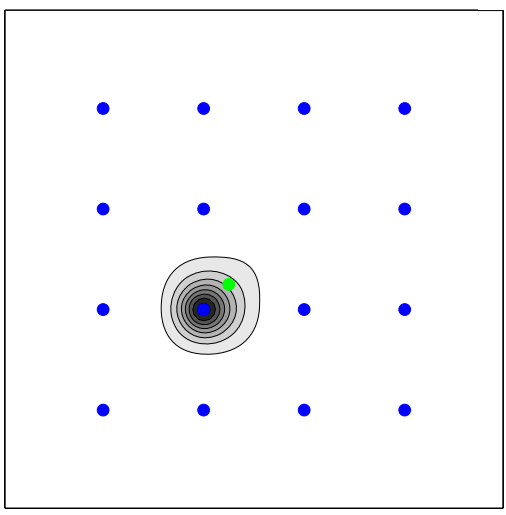

Fig. 4. The snapshot of the probability distribution for the non-parametric update algorithm after 10 seconds of computation.

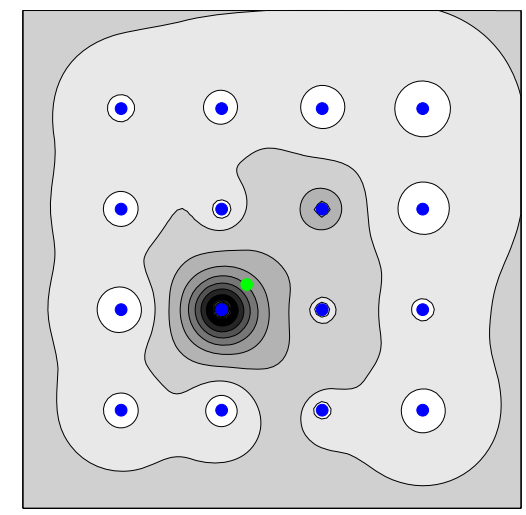

Fig. 5. The snapshot of the probability distribution for the bayesian update algorithm after two seconds of computation.

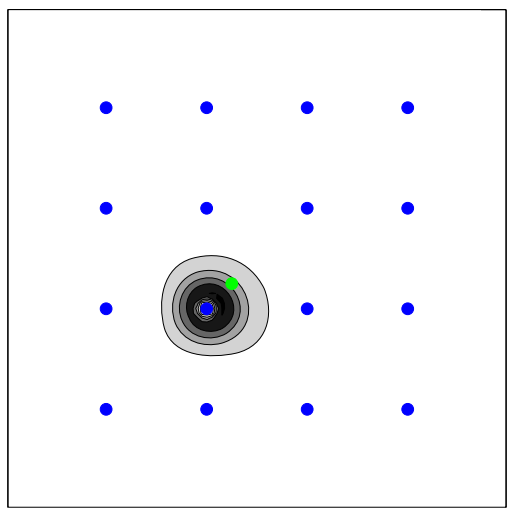

Fig. 6. The snapshot of the probability distribution for the bayesian update algorithm after 10 seconds of computation. 


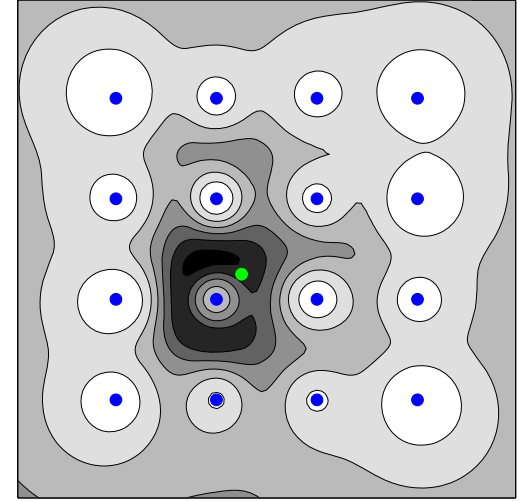

Fig. 7. The snapshot of the probability distribution for the non-parametric update algorithm after 2 seconds of computation in presence of noise.

per second.

Figures 7 and 9 show the posteriori distributions after 2 and 10 seconds, respectively, using the non-parametric Bayesian update strategy that does not use a priori distributions on source intensity. Figures 8 and 10 show the same two distributions using a Bayesian strategy that uses a prior distribution on source intensity - the same distribution as in the previous experiments. After 2 seconds the non-parametric strategy is slightly better since the a priori distribution we used is quite poor. After 10 seconds using priors on source intensity gives slightly better estimates, though both give adequate results.

Consider a region $\mathcal{A}$ of $100 \times 100$ squared meters centered around the agent positioned at coordinate $(200,200)$. The probability that the source is in $\mathcal{A}$ is estimated to be 0.36 and 0.58 after 2 and 10 seconds (respectively) for the algorithm that doesn't use priors on source intensity. The corresponding results for the algorithm that uses priors on intensity are 0.5 and 0.59 respectively.

Using a prior on source intensity where the prior assigns low probability to source rates near the background rates helps in distinguishing the background from a source. Also, when a prior on source intensity is not used the posteriori distributions assign some (low) probability to regions far from the true source because of the background radiation received by sensors far from the source.

\section{Exploiting Directionality}

In some situations, light poles or telegraph poles, are far apart in a field and it is not possible to place temporary traffic signs on which sensors can be located. A solution is to place multiple sensors on a pole. The sensors are crystals that can be analyzed as thin sheets that allow only photons from one direction because the reverse direction is shielded by metal. We first study a system in which 8 sensors are placed on each pole in an octagon. The results are shown in figures 11 and 12. The algorithm without priors on source intensity

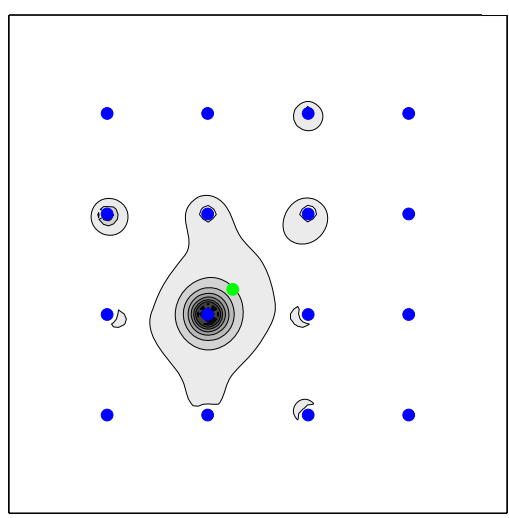

Fig. 8. The snapshot of the probability distribution for the bayesian update algorithm after 2 seconds of computation in presence of noise.

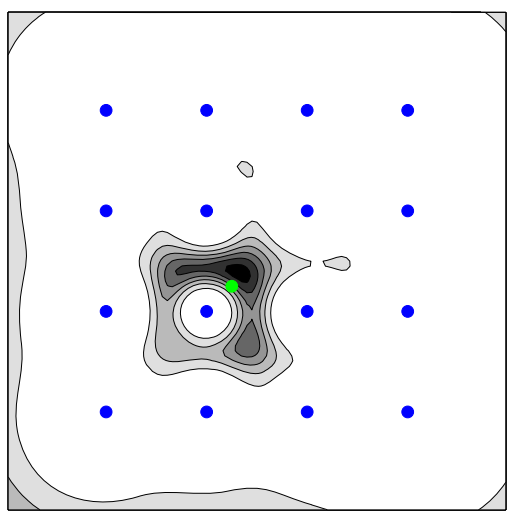

Fig. 9. The snapshot of the probability distribution for the non-parametric update after 10 seconds of computation in presence of noise.

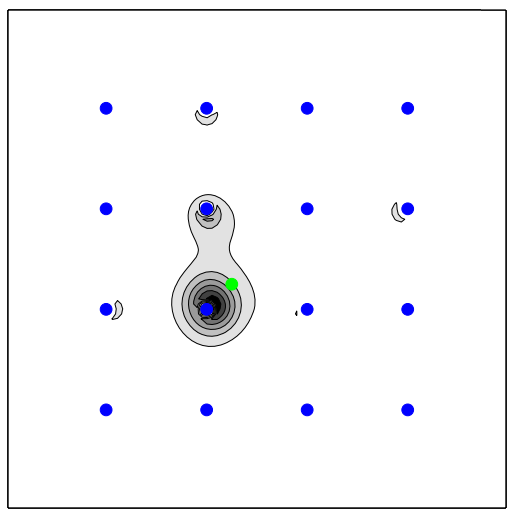

Fig. 10. The snapshot of the probability distribution for bayesian update algorithm after 10 seconds of computation in presence of noise. 


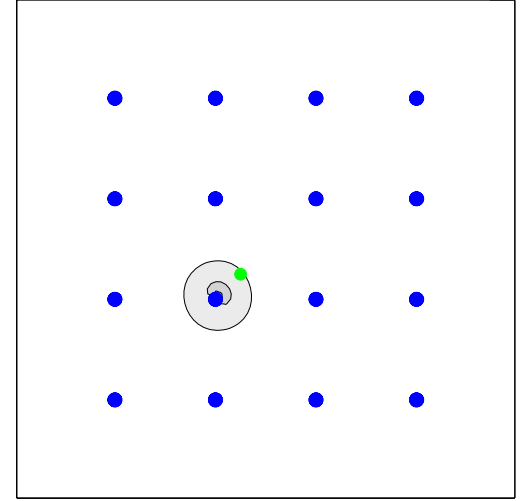

Fig. 11. The snapshot of the probability distribution for the non-parametric update after 2 seconds of computation when directionality is exploited and sensors are placed on each pole in an octagon.

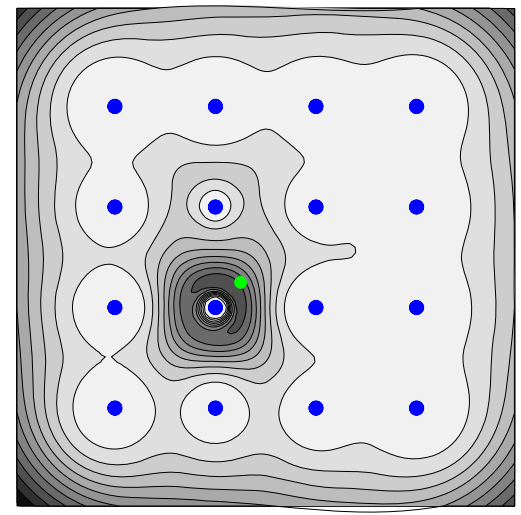

Fig. 12. The snapshot of the probability distribution for the bayesian update after 2 seconds of computation when directionality is exploited and sensors are placed on each pole in an octagon.

gives better results than the algorithm with priors because the prior distribution is poor. Let $\mathcal{A}$ be a circle centered at $(200,200)$ with radius $25 \mathrm{~m}$. This circle contains the source. The algorithm without priors assigns a probability of 0.87 that the source is in $\mathcal{A}$, whereas the algorithm with priors assigns only 0.41 probability. After 10 seconds, both algorithms are very accurate assigning a probability of 0.98 of that the source is (correctly) located with a small circle (radius $5 \mathrm{~m}$ ). Then, we study the case when 4 sensors are placed on each pole in a square. Figures 13 and 14 show the posteriori distributions after 2 seconds of computation. The algorithm without priors assigns a probability of 0.61 that the source is in $\mathcal{A}$, whereas the algorithm with priors assigns only 0.28 probability.

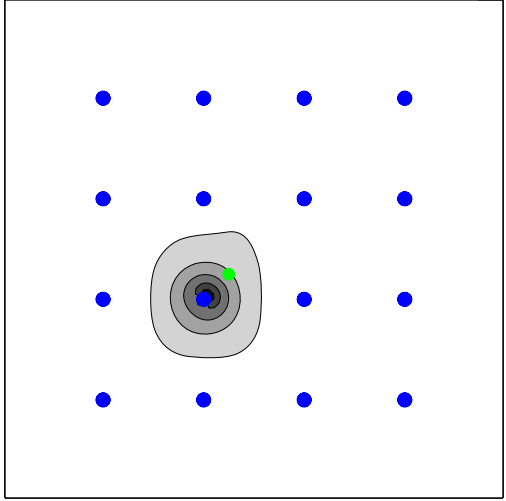

Fig. 13. The snapshot of the probability distribution for the non-parametric update after 2 seconds of computation when directionality is exploited and sensors are placed on each pole in an square.

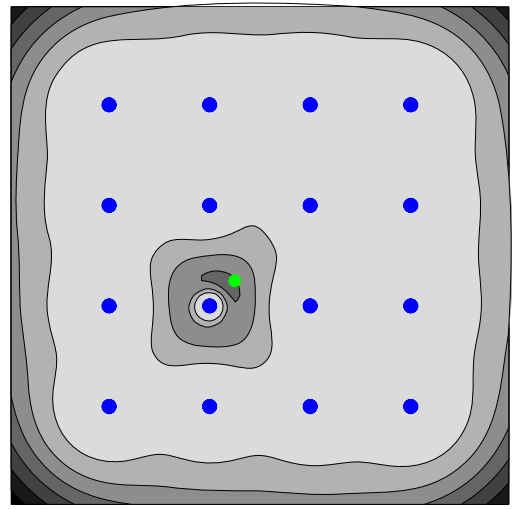

Fig. 14. The snapshot of the probability distribution for the bayesian update after 2 seconds of computation when directionality is exploited and sensors are placed on each pole in an square.

\section{CONCLUSION AND FURTHER WORK}

This study shows that networks of static sensors can help direct security personnel towards radiation sources rapidly. Typically, security personnel will also carry sensors and they can verify that the target is a true source of radiation by moving close to a target. Further by using sensors that detect isotope signatures they can reduce rates of false positives by differentiating medical radiation and other innocuous sources from criminals.

What should security personnel look out for? The time to detect and locate a source is inversely proportional to the rate at which the source generates photons. Therefore, a criminal may attempt to reduce this rate by shielding the source with lead or other metal. Reducing the source rate by a factor of 100 increases the time for the sensor network to detect 
and locate the source by a factor of 100; thus instead of merely 10 seconds the network will require 16 minutes. In this case a moving source can evade detection. The amount of lead required to reduce radiation from a high energy source, such as Cesium-137, by a factor of 100 cannot be carried conveniently. Therefore, the sensor network will have the most difficulty detecting criminals with metal-shielded sources carried in wheeled luggage or some type of vehicle.

The results show that directional sensors detect and locate sources rapidly provided there are enough sensors. This paper only reports on the benefits of plating sensors with metal on one surface so that the sensor only detects photons hitting the other surface. Better directionality through the use of sufficient numbers of collimators will provide even greater accuracy. Further research needs to be carried out on the tradeoffs between (a) greater numbers of more directional collimators on fewer utility poles and (b) less directional sensors on more utility poles that are closer together.

Our results show that algorithms that do not assume a prior on source intensities are more accurate after measurements over small durations than algorithms that assume poor priors; however, algorithms that do use priors are better over longer time spans. This suggests further research in which both algorithms are run concurrently.

The results in this paper suggest that networks of mobile sensors that can move towards targets will be more effective. The payload of a sensor coupled with wireless communication is small enough that it can be carried by very small UAVs, such as model airplanes, that fly quite close to the ground. The optimal search strategy for such mobile sensors is a subject of research.

\section{RELATED WORK}

Sensor networks consisting of large number of devices, each capable of some limited computation, communication and sensing [10], have been used in a wide variety of contexts, from battlefield surveillance to environmental monitoring [3], [8]. Energy efficient protocols for these networks have been extensively studied. More recently, Arora [1] uses geometric ideas to design an energy-efficient protocol which return the location of a particular object in response to an in-network subscriber issuing a find query regarding that object.

We employ a maximum likelihood estimator for estimating the position of the radiation source using the assumption that the source emits photons at a Poisson rate. Maximum likelihood estimation procedures have been used in different contexts, for example in target tracking to estimate the position of an unknown object using binary detection information sent by proximity sensors [9], or the kinematical coordinates of multiple targets in dynamic scenarios consisting of actual targets and false alarms [2].

Search strategies have been studied intensively at least since World War II ([5], [6] and [7]). Many of the problems studied in the literature deal with mobile searchers and evaders where searchers can "see" evaders when they are within a specified distance $D$. Our paper deals only with how networking static sensors can help with detection. The probabilistic nature and the physics of radiation offers new challenges.

\section{ACKNOWLEDGMENT}

This work is supported by AFOSR MURI award FA955006-1-0303 and the Lee Center for Networking.

\section{REFERENCES}

[1] V. Kulathumani, A. Arora, M. Demirbas and M. Sridharan. "Trail: A distance sensitive network protocol for distributed object tracking". In Proceedings of the European Conference on Wireless Sensor Networks (EWSN), 2007.

[2] Y. Bar-Shalom and T. E. Fortmann. "Tracking and Data Association". Academic Press, 1988.

[3] A. Cerpa, J. Elson, D. Estrin, L. Girod, M. Hamilton and J. Zhao. "Habitat monitoring: application driver for wireless communications technology". In Proceedings of the 2001 ACM SIGCOMM Workshop on Data Communications in Latin America and the Caribbean, San Jose, Costa Rica, pp. 20-41, 2001.

[4] E. Shih, S.-H. Cho, N. Ickes, R. Min, A. Sinha, A. Wang, and A. Chandrakasan. "Physical layer driven algorithm and protocol design for energy-efficient wireless sensor networks". In Proceedings of the 7th Annual ACM/IEEE International Conference on Mobile Computing and Networking (MobiCom), Italy, pp. 272287, 2001.

[5] D.E. Cullen, B.A. Knoppers, Z.F. Lansdowne and A.J. Truelove. "Mathematical Research in Destroyer-Submarine Encounters.” Technical Report at the Defense Technical Information Center, number AD0730514, 1971.

[6] S. Lalley and H. Robbins. "Asymptotically Minimax Search Strategies in the Plane". In Proceedings National Academy of Sciences, 1987.

[7] T.G. McGee and J.K. Hedrick. "Guaranteed Strategies to Search for Mobile Evaders in the Plane". In Proceedings of the 2006 American Control Conference, Minneapolis, USA, 2006.

[8] A. Mainwaring, J. Polastre, R. Szewczyk, D. Culler and J. Anderson. "Wireless sensor networks for habitat monitoring". In Proceedings of the 1st ACM International Workshop on Wireless Sensor Networks and Application (WSNA02), Atlanta, USA, pp. 88-97, 2002.

[9] Rodriguez, M. Lazaro, and L. Tong. "Target Location Estimation in Sensor Networks using Range Information". In Prooceedings of the IEEE Sensor Array and Multichannel Signal Processing workshop, Sitges, Spain, 2004.

[10] F. Zhao and L. Guibas. "Wireless sensor networks: an information processing approach”. Morgan Kaufmann Publishers, 2004. 